\title{
A Comparison of Biannual Two-Phase Low- Dose Liver CT and US for HCC Surveillance in a Group at High Risk of HCC Development
}

\author{
Jeong Hee Yoon ${ }^{a, b}$ Jeong Min Lee ${ }^{a-c}$ Dong Ho Lee ${ }^{a, b}$ ljin Joo ${ }^{a, b}$ \\ Ju Hyun Jeon ${ }^{a, b}$ Su Joa Ahn ${ }^{a, b}$ Seung-taek Kim ${ }^{a, b}$ Eun Ju Cho ${ }^{d}$ \\ Jeong-Hoon Lee ${ }^{d}$ Su Jong Yu ${ }^{d}$ Yoon Jun Kim ${ }^{d}$ Jung-Hwan Yoon ${ }^{d}$ \\ ${ }^{a}$ Radiology, Seoul National University Hospital, Seoul, Republic of Korea; ${ }^{b}$ College of \\ Medicine, Seoul, Republic of Korea; ' Institute of Radiation Medicine, Seoul National \\ University Medical Research Center, Seoul, Republic of Korea; ${ }^{\mathrm{I} I n t e r n a l ~ M e d i c i n e ~ a n d ~ L i v e r ~}$ \\ Research Institute, Seoul National University College of Medicine, Seoul, Republic of Korea
}

\section{Keywords}

Hepatocellular carcinoma · Surveillance · Ultrasonography · Low-dose CT · Cirrhosis

\begin{abstract}
Background and Aims: Biannual ultrasonography (US) is a current recommendation for hepatocellular carcinoma (HCC) surveillance in a high-risk group. The sensitivity of US, however, has been low in patients with a high risk of developing HCC. We aimed to compare sensitivity for HCC of biannual US and two-phase low-dose computed tomography (LDCT) in patients with a high risk of HCC. Methods: In this prospective single-arm study, participants with an annual risk of HCC greater than $5 \%$ (based on a risk index of $\geq 2.33$ ) and who did not have a history of HCC were enrolled from November 2014 to July 2016. Participants underwent paired biannual US and two-phase LDCT 1-3 times. Two-phase LDCT included arterial and 3-min delayed phases. The sensitivity, specificity, and positive predictive value of HCC detection using US and two-phase LDCT were compared using a composite algorithm as a standard of reference. Results: Of the 139 enrolled participants, 137 underwent both the biannual US and twophase LDCT at least once and had follow-up images. Among them, 27 cases of HCC (mean size: $14 \pm 4 \mathrm{~mm}$ ) developed in 24 participants over 1.5 years. Two-phase LDCT showed a significantly higher sensitivity (83.3\% [20/24] vs. $29.2 \%[7 / 24], p<0.001)$ and specificity $(95.6 \%$ [108/113] vs. 87.7\% [99/113], $p=0.03$ ) than US. A false-positive result was reported in 14 participants at US and 5 participants at two-phase LDCT, resulting in a significantly higher posi-
\end{abstract}


Yoon et al.: HCC Surveillance Using Biannual Two-Phase LDCT and US in a High-Risk Group

tive predictive value of two-phase LDCT (33.3\% [7/21] vs. 80\% [20/25], $p<0.001)$. Conclusions: Patients with a risk index $\geq 2.33$ showed a high annual incidence of HCC development in our study, and two-phase LDCT showed significantly higher sensitivity and specificity for HCC detection than US.

(C) 2020 The Author(s)

Published by S. Karger AG, Basel

\section{Introduction}

Primary liver cancer was the sixth most common malignancy worldwide in 2018, and hepatocellular carcinoma (HCC) accounts for $75-85 \%$ of primary liver cancer [1], with an increasing incidence in Europe and the USA [2]. The prognosis of patients with HCC is quite poor, with a reported 5-year survival rate $<20 \%[3,4]$. The mortality rate for HCC has also risen in Europe as well as North and South America with geographic variation [5]; and while it has decreased in Asia, there is still a 2- to 5-fold higher mortality rate there than in other continents [5]. Because the prognosis is related to the extent of the disease at the time of diagnosis, regular surveillance of high-risk groups is recommended to detect early-stage cancer [6].

Currently, biannual ultrasonography (US) is recommended by major guidelines for the surveillance of patients at risk of HCC [7-9]. The sensitivity of US, however, has been reported as only $27.9-63 \%$ for early-stage HCC detection [10-12]. Even though they are most at risk of developing HCC, its detection via US is often especially challenging for patients with advanced liver cirrhosis - possibly due to the limited sonic window and multiple cirrhotic nodules [12-14]. In recent years, studies have investigated the feasibility of gadoxetic acidenhanced magnetic resonance imaging (MRI), computed tomography (CT), or contrastenhanced US (CEUS) for HCC surveillance as an alternative to US [15-18].

Although CT has been known to show higher sensitivity for the detection of HCC than US $[14,16]$, it has not been recommended for HCC surveillance owing to the cost and concerns about radiation exposure. However, the cost-effectiveness of cancer screening is heavily dependent on the prevalence of cancer and the sensitivity of the screening test [19]. Although risk stratification of HCC development in cirrhotic patients with diverse causes has not yet been fully developed, if CT can provide significantly higher sensitivity than US while using a reasonably low radiation dose, the use of CT can be justified in a population with a high risk of developing HCC. Recently, several CT techniques for radiation dose reduction - including automatic current modulation, automatic peak kilovoltage $(\mathrm{kVp})$ selection, and iterative reconstruction algorithms - have been implemented widely, which has contributed to a reduction in radiation exposure without hampering image quality and diagnostic performance $[20,21]$. Moreover, in a surveillance setting, we may not need four-phase liver CT which is a standard protocol for diagnostic liver CT examination [22].

Thus, the primary purpose of our study is to investigate the clinical feasibility of twophase low-dose CT (LDCT) as a surveillance imaging modality in a group at high risk of HCC development.

\section{Materials and Methods}

Study Enrollment

Between November 2014 and July 2016, 139 participants were prospectively enrolled according to the following eligibility criteria: participants had to (a) be at least 20 years old; (b) have chronic hepatitis B or cirrhosis of any etiology that is considered part of a group at high risk of developing HCC according to the American Association for the Study of Liver Diseases (AASLD) guidelines [23]; (c) be currently on regular biannual surveillance using US; (d) have a risk index $\geq 2.33$, which correlates to an annual HCC incidence of 
Yoon et al.: HCC Surveillance Using Biannual Two-Phase LDCT and US in a High-Risk Group

more than 5\% [24]; and (e) have provided signed informed consent. Exclusion criteria were as follows: participants (a) were previously diagnosed with HCC; (b) had any contraindication of contrast-enhanced CT; and (c) had any history of other malignancies. The risk index of inclusion criterion was calculated using the following equation:

Risk index $=1.65$ (if the prothrombin activity is $\leq 75 \%$ ) +1.41 (if the age is $\geq 50$ years) +0.92 (if the platelet count is $\leq 100 \times 10^{3} / \mathrm{mm}^{3}$ ) +0.74 (if anti-hepatitis C virus antibody or hepatitis B surface antigen is positive) [24].

The absence of HCC was determined via US or CT over the 6 months prior to enrollment. We did not exclude Child B or C patients.

Study Protocol

Enrolled participants underwent paired US and two-phase contrast-enhanced LDCT 1-3 times at 6-month intervals. The interval between paired US and LDCT was kept within 3 weeks. During the study, participants were censored from the study if HCC was detected, and referred to the standard of care.

Image Acquisition

US Examination

Participants were requested to fast for $6 \mathrm{~h}$. Subsequently, US was performed by one of our fellowshiptrained board-certified radiologists (J.H.Y., J.M.L., D.H.L., I.J., J.H.J., S.J.A., S.K.) with the ultrasound scanners using a convex probe (2-5 MHz). The scan included images of an axial and longitudinal view of the left hemiliver in addition to a subcostal/intercostal view of the right hemiliver.

Two-Phase LDCT

CT was performed with a dedicated scanner (Discovery; GE Healthcare, Milwaukee, WI, USA). Twophase (arterial, 3-min delayed phase) CT was obtained using iodine contrast media $(1.5 \mathrm{~mL} / \mathrm{kg}$, Iobrix 350 , Acuzen, Seoul, Korea, or Optiray 350, Reyon Pharma, Seoul, Korea). Scan coverage was from the basal lung to the iliac crest. Depending on patients' weight, $80 \mathrm{kVp}$ (body weight $\leq 70 \mathrm{~kg}$ ) or $100 \mathrm{kVp}$ (body weight $>70$ $\mathrm{kg}$ ) was applied. The dose-length product was automatically calculated, and the effective dose was estimated using a coefficient of 0.015 [25].

\section{Image Interpretation}

A report for the US was made by radiologists in each imaging session. Radiologists who performed US were blind to the results from the CT scan at the same round, but prior CT images were open to them. Likewise, the CT reports were made by radiologists kept blind to the results of the US performed at the same round. Detected observations were recorded in terms of their size, location, echogenicity (at US), and enhancement pattern (two-phase LDCT). For US, a positive report for HCC was defined as when observation with suspicion of HCC ( $\geq 1 \mathrm{~cm}$ solid nodule except known benign nodules without interval change, or $<1 \mathrm{~cm}$ with peripheral halo, typical mosaic pattern, or newly developed distinctive nodule) or thrombus was detected with a recommendation for further examination other than a 6-month regular US follow-up. CT or MRI recommendation due to a poor sonic window or multiple small nodules was not regarded as a surveillance-positive report.

In the case of two-phase LDCT reporting, only observations with Liver Imaging Reporting and Data System (LI-RADS) scoring 3 or higher using LI-RADS v2014 [26] were reported. At two-phase LDCT, a positive report for HCC was defined as when observations with LR-4 or LR-5 existed or observations of LR-3 with a recommendation for further examination other than a 6-month follow-up.

\section{Standard of Reference}

In participants with suspicious hepatic observations, standard-dose four-phase liver CT or gadoxetic acid-enhanced liver MRI was recommended. In participants without detected hepatic observations during the study period, absence or presence of HCC was determined by follow-up imaging in the $6-12$ months following the last two-phase LDCT. Diagnosis of HCC was made via a composite algorithm using either pathologic results or typical imaging features on cross-sectional imaging. We considered observations featuring LR-4 with arterial phase hyperenhancement (APHE), LR-5, and LR-5V on standard dose four-phase liver CT or gadoxetic acid-enhanced MRI as HCC. If the observation was consistent with LR-4, LR-5, or LR-5V at two- 
Table 1. Demographics of the study participants

\begin{tabular}{|c|c|}
\hline Variables & Values \\
\hline Sex (men:women), $n$ & $96: 42$ \\
\hline \multicolumn{2}{|l|}{ Age, years } \\
\hline Men & $58 \pm 7.1(41-77)$ \\
\hline Women & $62.2 \pm 8.7(40-82)$ \\
\hline BMI & $24.6 \pm 2.9(17.5-34.8)$ \\
\hline $\mathrm{BMI}<25: \mathrm{BMI} \geq 25, n$ & $87: 50$ \\
\hline \multicolumn{2}{|l|}{ Underlying disease, $\%(n / N)$} \\
\hline Chronic hepatitis B & $83.3(115 / 138)$ \\
\hline Chronic hepatitis C & $2.2(3 / 138)$ \\
\hline Alcoholic liver disease & $2.9(4 / 138)$ \\
\hline Hepatitis B and alcoholic & $6.5(9 / 138)$ \\
\hline Cryptogenic & $1.4(2 / 138)$ \\
\hline Autoimmune hepatitis & $0.7(1 / 138)$ \\
\hline Budd-Chiari syndrome & $0.7(1 / 138)$ \\
\hline Cardiac cirrhosis & $1.4(2 / 138)$ \\
\hline Wilson's disease & $0.7(1 / 138)$ \\
\hline \multicolumn{2}{|l|}{ Laboratory findings } \\
\hline Albumin, g/dL & $3.9 \pm 0.5(2.2-5.0)$ \\
\hline Total bilirubin, mg/dL & $2.0 \pm 1.2(0.4-5.9)$ \\
\hline Prothrombin time, INR & $1.23 \pm 0.28(0.98-3.54)$ \\
\hline Platelet count, $\times 10^{3} / \mathrm{mm}^{3}$ & $68 \pm 39(7-280)$ \\
\hline $\mathrm{AFP}, \mathrm{ng} / \mathrm{mL}$ & $4.6 \pm 7.5(1-80.2)$ \\
\hline $\mathrm{AFP} \leq 20 \mathrm{ng} / \mathrm{mL}, n$ & 133 \\
\hline $\mathrm{AFP}>20 \mathrm{ng} / \mathrm{mL}, n$ & 4 \\
\hline Creatinine, $\mathrm{mg} / \mathrm{dL}$ & $0.8 \pm 0.2(0.4-1.37)$ \\
\hline Risk index & $3.41 \pm 0.75(2.33-4.72)$ \\
\hline \multicolumn{2}{|l|}{ Child-Pugh class, $\%(n / N)$} \\
\hline Class A & $71.7(99 / 138)$ \\
\hline Class B & $26.8(37 / 138)$ \\
\hline Class C & $1.5(2 / 138)$ \\
\hline Antiviral agent (Yes:No), $n$ & $82: 56$ \\
\hline \multicolumn{2}{|l|}{ Radiation dose } \\
\hline $100 \mathrm{kVp}: 80 \mathrm{kVp}, n$ & $46: 92$ \\
\hline Dose-length product, mGy & $527.8 \pm 200.5(153.2-1113.5)$ \\
\hline Effective dose, $\mathrm{mSv}$ & $7.9 \pm 3.0(2.3-17.3)$ \\
\hline
\end{tabular}

Values are mean \pm standard deviation (range), $n$, or $\%(n / N)$, as appropriate. AFP, alpha-fetoprotein; BMI, body mass index; INR, international normalized ratio.

phase LDCT and showed tumor staining on cone-beam CT for transarterial chemoembolization (TACE) followed by compact lipiodol uptake on follow up noncontrast CT, we also classified them as HCC.

\section{Outcome}

The primary end point was to compare HCC detection rates for biannual US and two-phase LDCT, regardless of the stage. Secondary end points included comparisons of very early/early-stage HCC detection rates and false-positive rates for the two surveillance modalities. According to the Barcelona Clinic Liver Cancer criteria [27], very early-stage HCC is defined as a single nodule smaller than $2 \mathrm{~cm}$, and early-stage HCC as a single lesion larger than $2 \mathrm{~cm}$ or 2 to 3 lesions each smaller than $3 \mathrm{~cm}$. Stage was determined based only on tumor size and number in our study.

\section{Statistical Analysis}

Comparison of HCC detection between US and two-phase LDCT was made using generalized estimating equation analysis considering subject correlation. For comparisons of variables among participants with or without HCC development, an independent $t$ test was used for continuous variables and either a $\chi^{2}$ test or 


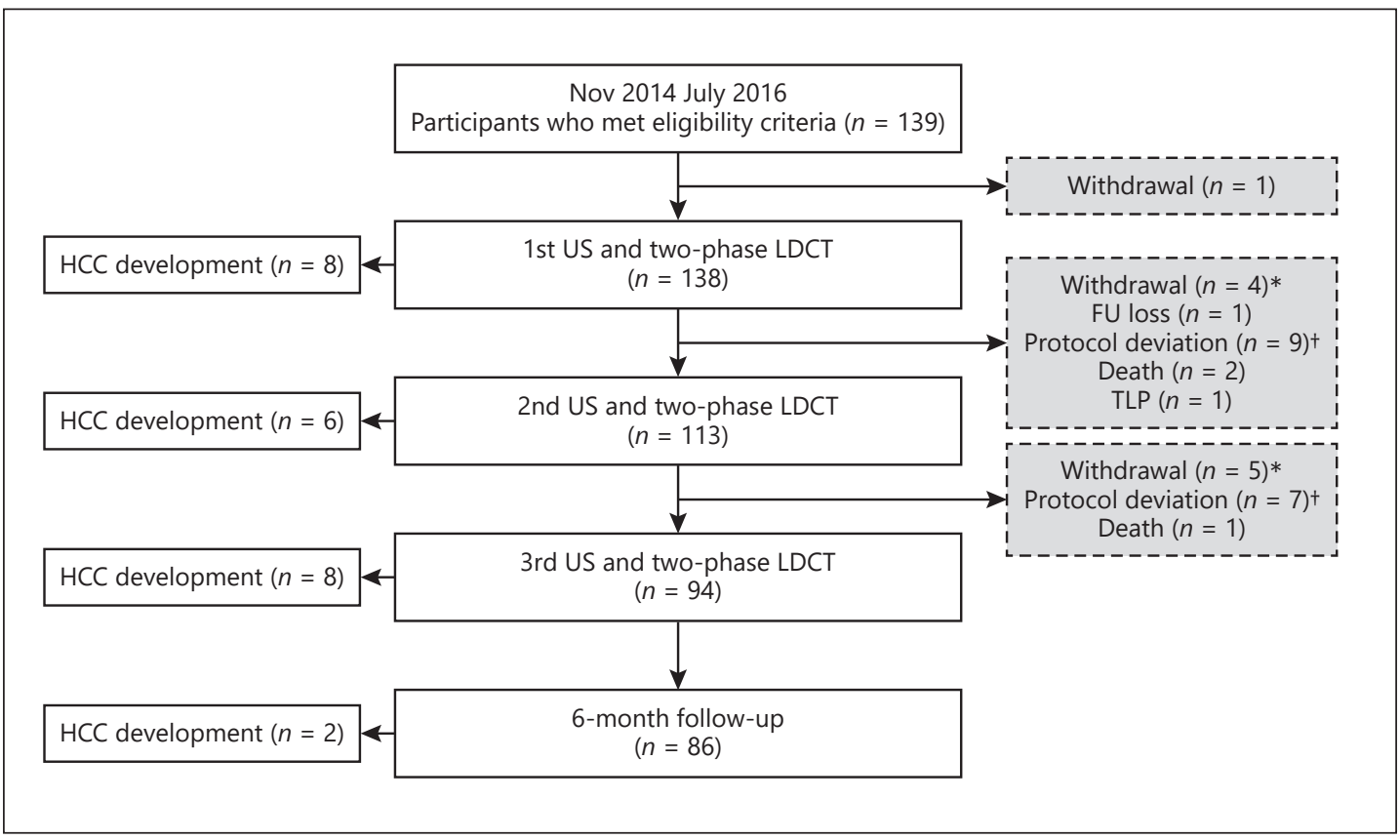

Fig. 1. Study flow. * Participants had follow-up examinations before the withdrawal. ${ }^{\dagger}$ Long interval between each round (>9 months). CT, computed tomography; FU, follow-up; HCC, hepatocellular carcinoma; LDCT, low dose CT; TPL, transplantation; US, ultrasonography.

Fisher's exact test was used for categorical variables. All statistical analyses were performed using commercially available software packages (IBM SPSS version 25, SPSS Inc., IBM Company, Armonk, NY, USA; Medcalc version 14.12.0, Medcalc Software, Ostend, Belgium; SAS version 9.4, SAS Institute Inc, Cary, NC, USA; and PASS version 15, NCSS LLC., Kaysville, Utah, USA). A $p$ value of less than 0.05 was considered to indicate a significant difference.

\section{Results}

Of the 139 participants enrolled in the study, 138 (men:women =96:42) underwent the first round of US and two-phase LDCT. The median interval between US and two-phase LDCT was 0 days (range 0-24 days). Detailed demographics are displayed in Table 1 . During the follow-up period, 30 participants were dropped due to withdrawal $(n=9)$, loss to follow-up ( $n=1)$, protocol deviation due to study interval ( $>9$ months) between the rounds $(n=16)$, death resulting from hepatic failure $(n=3)$, or liver transplantation $(n=1)$ (Fig. 1). In 86 participants with a negative finding in the last pairing of US and two-phase LDCT, the presence or absence of HCC after study completion was determined via at least 6-month follow-up imaging using either US $(n=65)$, standard-dose four-phase liver CT $(n=18)$, or gadoxetic acid-enhanced MRI $(n=3)$. The analysis was performed for 137 participants (men:women = 95:42) with follow-up imaging after the first round of US and two-phase LDCT, after excluding a man without follow-up imaging. The median follow-up interval was 17 months (range 0-31 months) from the first round to final diagnosis.

\section{Incidence of HCC Development in Study Participants}

A total of 27 HCCs were found in 24 participants over 1.5 years (Fig. 1), which suggests an annual incidence of $11.7 \%$ for HCC development. Twenty-one participants had a single 


\section{Liver Cancer}
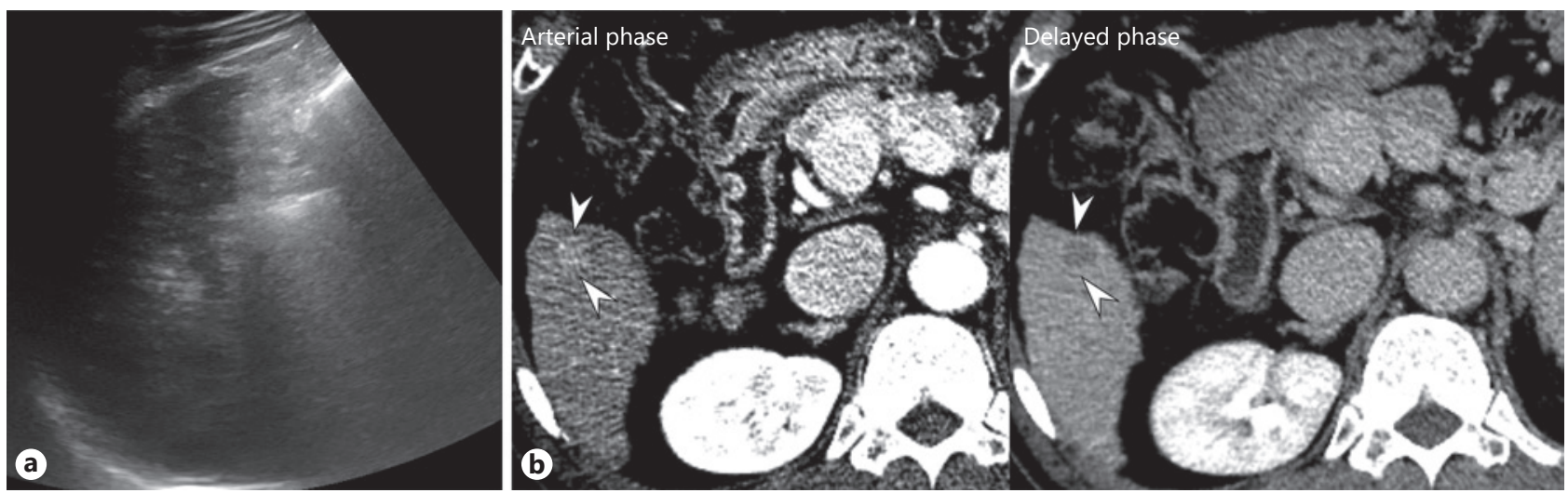

Fig. 2. A 58-year-old man with HCC. a No focal hepatic observation was reported at US. b Approximately 15mm observation with arterial phase hyperenhancement (APHE) and delayed washout is seen at two-phase LDCT (arrowheads). The observation showed APHE and portal washout on gadoxetic acid-enhanced MRI and was clinically diagnosed as an HCC.

Table 2. Comparison of the demographics of participants with and without HCC development

\begin{tabular}{lccc}
\hline & $\begin{array}{l}\text { No HCC development } \\
(n=113)\end{array}$ & $\begin{array}{l}\text { HCC development } \\
(n=24)\end{array}$ & $\begin{array}{l}p \\
\text { value }\end{array}$ \\
\hline Sex (men:women), $n$ & $76: 37$ & $19: 5$ & 0.33 \\
Age, years & $59 \pm 8(40-77)$ & $61 \pm 7(50-78)$ & 0.42 \\
Risk index & $3.35 \pm 0.71(2.33-4.72)$ & $3.75 \pm 0.85(2.33-4.72)$ & 0.02 \\
Child-Pugh (A:B or C), $n$ & $85: 28$ & $14: 10$ & 0.15 \\
Underlying disease (viral ${ }^{\mathrm{a}}$ :nonviral) & $103: 10$ & $23: 1$ & 0.72 \\
Antiviral agent (Yes:No) ${ }^{\mathrm{b}}, n$ & $62: 41$ & $19: 4$ & 0.07 \\
Body mass index $_{\text {AFP levelc }}$, ng/mL & $24.3 \pm 2.8(17.5-34.6)$ & $25.5 \pm 3.2(21.5-34.8)$ & 0.07 \\
AFP $\leq 20 \mathrm{ng} / \mathrm{mL}: \mathrm{AFP}>20 \mathrm{ng} / \mathrm{mL}), n$ & $3.7 \pm 3.8(1.0-27.5)$ & $11.7 \pm 21.3(1.9-80.2)$ & $<0.001$ \\
\hline
\end{tabular}

Values are mean \pm standard deviation (range) unless otherwise indicated. AFP, alpha-fetoprotein; HCC,

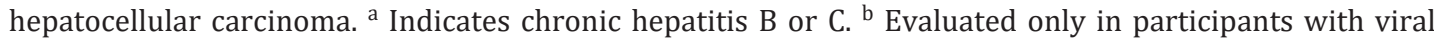
hepatitis $(n=126) .{ }^{c}$ Comparison between AFP level at HCC detection in HCC group and baseline AFP level in non-HCC group.

tumor, and 3 had two tumors. The average tumor size was $14 \pm 4 \mathrm{~mm}$ (range 8-23 $\mathrm{mm}$ ). Among the 24 participants with HCC development, 17 had very early-stage HCC and 7 had early-stage HCC. None of the participants had intermediate- or advanced-stage HCC. The HCCs were discovered in both the left lobe $(n=4)$ and the right lobe $(n=23)$. A diagnosis of HCC was made via the imaging features of gadoxetic acid-enhanced MRI $(n=17)$, standard-dose four-phase liver CT $(n=6)$, and observation with LR-4 at two-phase LDCT with tumor staining at cone-beam CT for TACE, compact lipiodol uptake at noncontrast CT, and marginal recurrence during the follow-up $(n=1)$. Participants with HCC development showed a significantly higher risk index than those without HCC development (3.35 \pm 0.71 vs. $3.75 \pm 0.85, p=0.02$ ) and alpha-fetoprotein (AFP) level $(p<0.001)$. However, there were no significant differences in sex, age, Child-Pugh class, cause of cirrhosis, and the number of participants with elevated AFP level between the groups with and without HCC development ( $p>0.05$ for all; Table 2). In 2 participants with HCC and elevated AFP level, 

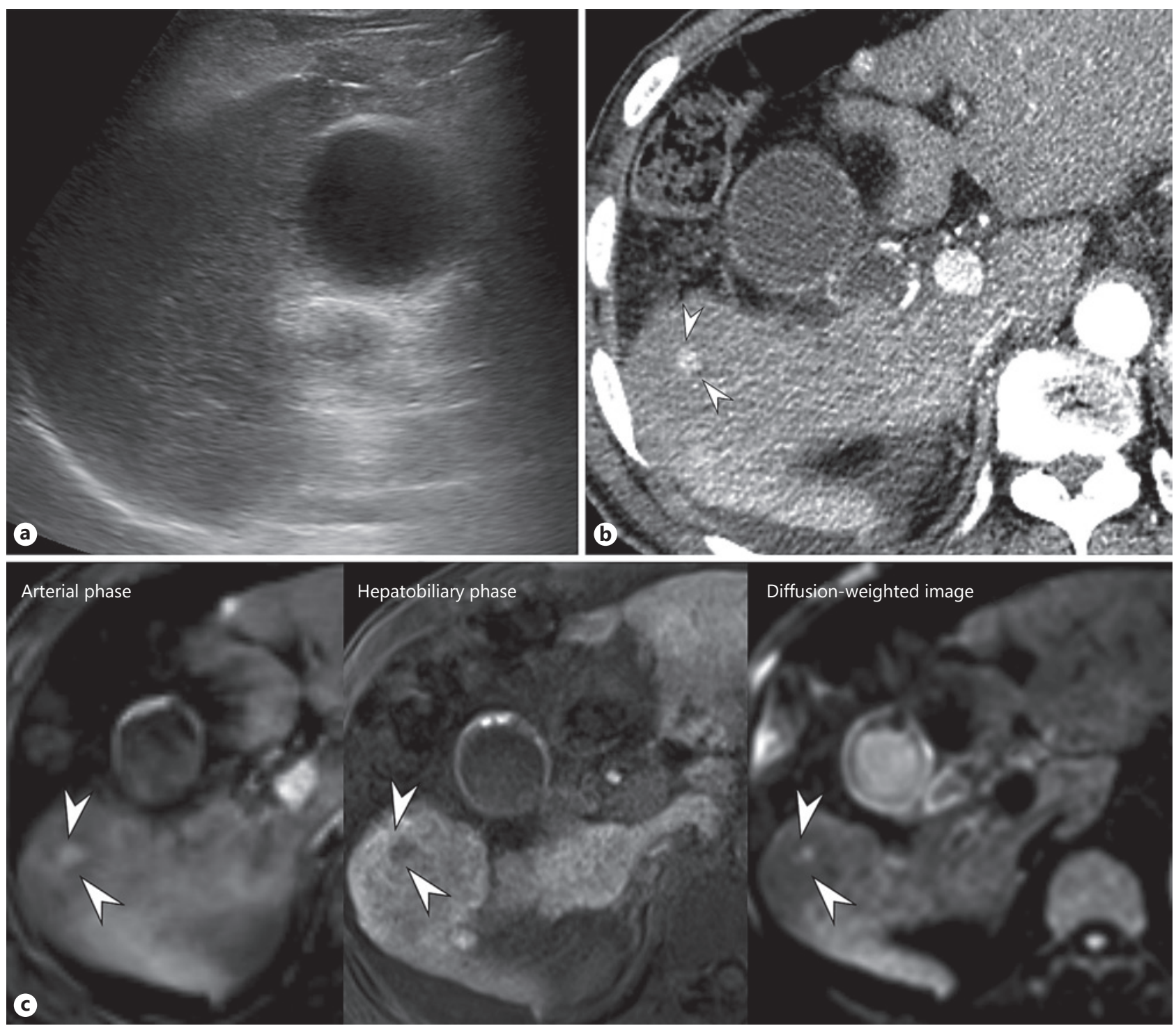

Fig. 3. A 62-year-old man with HCC. a At US, no focal hepatic observation was detected. b At two-phase LDCT, an approximately 10-mm observation with APHE is observed in segment 5 (arrowheads) without delayed washout (not shown). c At subsequent gadoxetic acid-enhanced MRI, the observation shows APHE, hepatobiliary phase defect, and diffusion restriction (arrowheads) and so is regarded as a probable HCC.

one participant had HCC at both US and two-phase LDCT whereas the other had no detected observation at US, and HCC was reported as arterioportal shunt at two-phase LDCT. Participants underwent radiofrequency ablation $(n=7)$, TACE $(n=14)$, transplantation after bridging therapy including ablation and TACE $(n=3)$.

\section{Comparison of per-Patient Sensitivity and Specificity for HCC Detection in the Two}

Protocols

In 24 participants diagnosed with HCC ( $n=27), 14$ HCCs in 13 participants were detected only at two-phase LDCT (mean tumor size: $12.7 \pm 3.7 \mathrm{~mm}$, range 8-22 mm) (Fig. 2, 3). Both two-phase LDCT and US reported the possibility of HCC in 7 participants with 9 HCCs (mean tumor size: $16.7 \pm 4.9 \mathrm{~mm}$, range $10-23 \mathrm{~mm}$ ): 9 and 7 HCCs were found at two-phase LDCT 

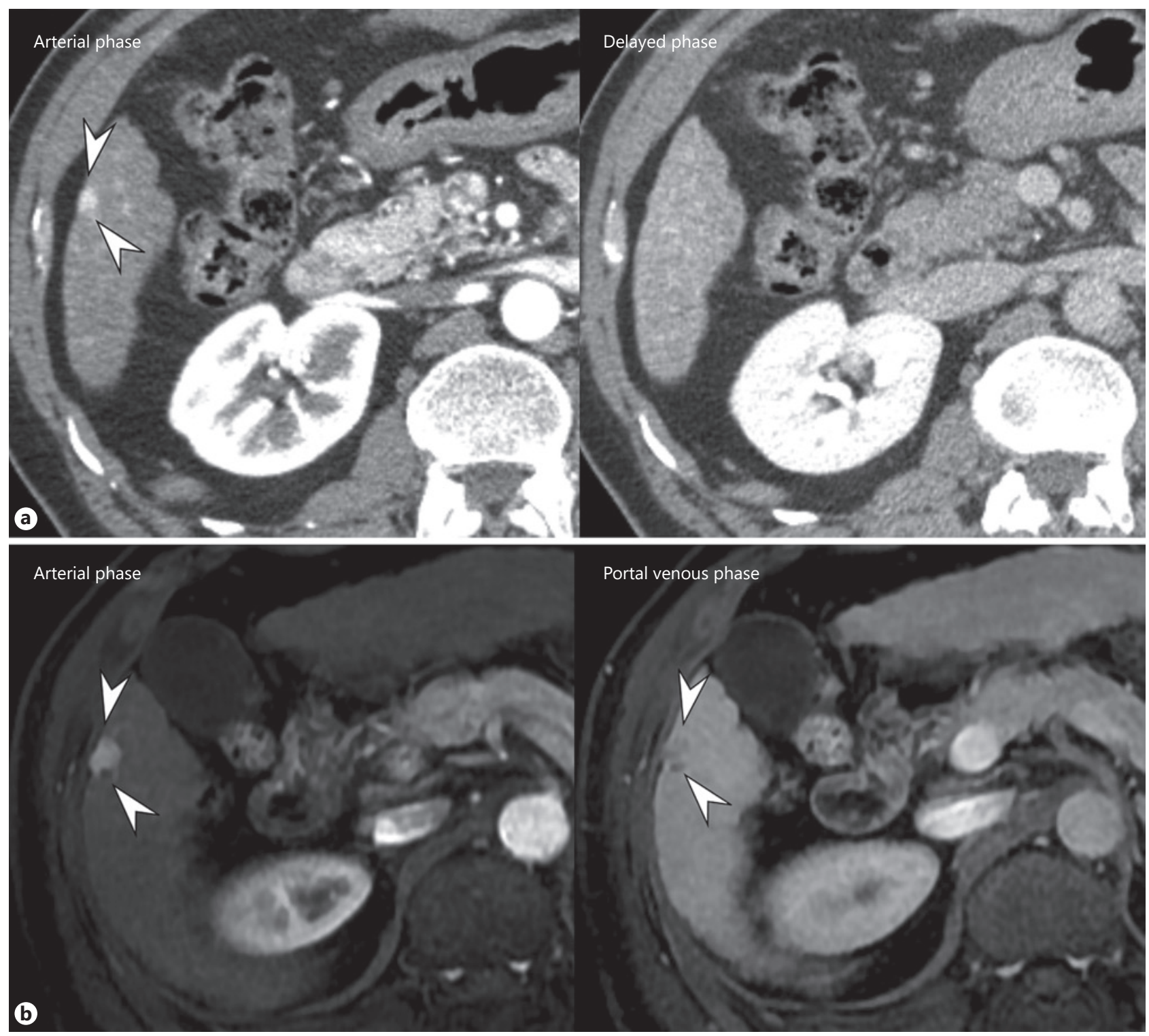

Fig. 4. A 53-year-old man with HCC. No focal hepatic observation was detected at US (not shown). a Approximately $8 \mathrm{~mm}$ observation with APHE is seen in the segment 6 subcapsular area (arrowheads) without delayed washout at two-phase LDCT. The observation was reported as an arterioportal shunt. b At follow-up gadoxetic acid-enhanced MRI, however, the observation $(14 \mathrm{~mm}$ ) shows APHE and portal washout (arrowheads) and so is regarded as HCC.

and US, respectively. Both missed 4 HCCs in 4 participants (mean tumor size: $15.3 \pm 3.6 \mathrm{~mm}$, range 10-20 mm) (Fig. 4). There were no participants with HCC found only at US. Two-phase LDCT showed a significantly higher sensitivity $(83.3 \%$ [20/24] vs. $29.2 \%$ [7/24], $p<0.001)$ and specificity (95.6\% [108/113] vs. 87.7\% [99/113], $p=0.03)$ than US (Table 3). Two-phase LDCT also had higher positive predictive value (PPV, 80\% [20/25] vs. 33.3\% [7/21], $p<$ 0.001 ) and negative predictive value (NPV, 96.4\% [108/112] vs. 85.3\% [99/116], $p<0.001$ ) than US (Table 3). Two-phase LDCT showed a significantly higher detection rate than US in both compensated and decompensated cirrhosis, participants with BMI $<25$, and those on antiviral medication (Table $4 ; p=0.004-0.03$ ). In participants with BMI $\geq 25$ and those without 
Table 3. Comparison of performance for HCC detection US and two-phase LDCT on a per-patient basis

\begin{tabular}{|c|c|c|c|c|c|c|}
\hline & \multicolumn{2}{|l|}{ Two-phase LDCT } & \multicolumn{2}{|l|}{ US } & \multirow{2}{*}{$\begin{array}{l}\text { Difference, } \\
\%(95 \% \mathrm{CI})\end{array}$} & \multirow{2}{*}{$\begin{array}{l}p \\
\text { value }\end{array}$} \\
\hline & $\begin{array}{l}\text { estimates, } \\
\%(n / N)\end{array}$ & $\begin{array}{l}95 \% \mathrm{CI}, \\
\%\end{array}$ & $\begin{array}{l}\text { estimates, } \\
\%(n / N)\end{array}$ & $\begin{array}{l}95 \% \mathrm{CI}, \\
\%\end{array}$ & & \\
\hline \multicolumn{7}{|c|}{ All HCCs $(n=24)$} \\
\hline Sensitivity & $83.3(20 / 24)$ & $63.1-93.6$ & $29.2(7 / 24)$ & $14.6-49.8$ & $54.2(34.2-74.1)$ & $<0.001$ \\
\hline Specificity & $95.6(108 / 113)$ & $89.9-98.2$ & $87.7(99 / 113)$ & $80.3-92.6$ & $8.0(1.0-15.0)$ & 0.03 \\
\hline PPV & $80(20 / 25)$ & $60-91.4$ & $33.3(7 / 21)$ & $16.8-55.3$ & $46.7(25.6-67.7)$ & $<0.001$ \\
\hline NPV & $96.5(108 / 112)$ & $91-98.7$ & $85.5(99 / 116)$ & $77.9-90.8$ & $11.1(5.4-16.7)$ & $<0.001$ \\
\hline \multicolumn{7}{|c|}{ Very early-stage HCC $(n=17)$} \\
\hline Sensitivity & $82.4(14 / 17)$ & $57.3-94.2$ & $17.7(3 / 17)$ & $5.8-42.7$ & $64.7(42.0-87.4)$ & $<0.001$ \\
\hline \multicolumn{7}{|c|}{ Early-stage of HCC $(n=7)$} \\
\hline Sensitivity & $85.7(6 / 7)$ & $41.9-98$ & $57.1(4 / 7)$ & $23-85.6$ & $28.6(-4.9$ to 62.0$)$ & 0.13 \\
\hline
\end{tabular}

US, ultrasonography; LDCT, low-dose computed tomography; CI, confidence interval; PPV, positive predictive value; NPV, negative predictive value.

Table 4. Comparison of performance for HCC detection between US and two-phase LDCT on a per-patient basis in subgroups

\begin{tabular}{|c|c|c|c|c|c|c|}
\hline & \multicolumn{2}{|c|}{ Two-phase LDCT } & \multicolumn{2}{|l|}{ US } & \multirow{2}{*}{$\begin{array}{l}\text { Difference, } \\
\%(95 \% \mathrm{CI})\end{array}$} & \multirow{2}{*}{$\begin{array}{l}p \\
\text { value }\end{array}$} \\
\hline & $\begin{array}{l}\text { estimates, } \\
\%(n / N)\end{array}$ & $\begin{array}{l}95 \% \mathrm{CI}, \\
\%\end{array}$ & $\begin{array}{l}\text { estimates, } \\
\%(n / N)\end{array}$ & $\begin{array}{l}95 \% \mathrm{CI}, \\
\%\end{array}$ & & \\
\hline \multicolumn{7}{|c|}{ Compensated cirrhosis $(n=99)$} \\
\hline Sensitivity & $71.4(10 / 14)$ & 42-90.4 & $28.5(4 / 14)$ & $9.5-58$ & $42.9(16.9-68.7)$ & 0.03 \\
\hline Specificity & $96.5(82 / 85)$ & $89.3-99.1$ & $83.5(71 / 85)$ & $73.6-90.4$ & $12.9(4.4-21.4)$ & 0.007 \\
\hline \multicolumn{7}{|c|}{ Decompensated LC $(n=38)$} \\
\hline Sensitivity & $100(10 / 10)$ & $65.5-100$ & $30(3 / 10)$ & $8.1-64.6$ & $70(41.6-98.4)$ & 0.02 \\
\hline Specificity & $92.9(2 / 28)$ & 75-98.8 & $100(28 / 28)$ & $85-100$ & $7.1(-2.4$ to 16.7$)$ & 0.5 \\
\hline \multicolumn{7}{|c|}{$\mathrm{BMI}<25(n=87)$} \\
\hline Sensitivity & $91.7(11 / 12)$ & $59.8-99.6$ & $16(2 / 12)$ & $9.4-25.9$ & $75(50.5-99.5)$ & 0.004 \\
\hline Specificity & $97.3(73 / 75)$ & $89.8-99.5$ & $84(63 / 75)$ & $74.1-90.6$ & $13.3(4.8-21.9)$ & 0.006 \\
\hline \multicolumn{7}{|c|}{$\mathrm{BMI} \geq 25(n=50)$} \\
\hline Sensitivity & $75(9 / 12)$ & $42.8-93.3$ & $41.7(5 / 12)$ & $16.5-71.4$ & $33.3(6.7-60)$ & 0.13 \\
\hline Specificity & $92.1(35 / 38)$ & $77.5-97.9$ & $94.7(36 / 38)$ & $81-99.1$ & $2.6(-8.8$ to 14.1$)$ & $>0.99$ \\
\hline \multicolumn{7}{|c|}{ With antiviral medication in viral hepatitis-related cirrhosis $(n=81)$} \\
\hline Sensitivity & $78.9(15 / 19)$ & $53.9-93$ & $31.6(6 / 19)$ & $8.3-52.6$ & $47.4(24.9-69.8)$ & 0.004 \\
\hline Specificity & $95.2(56 / 62)$ & $85.6-98.7$ & $83.9(52 / 62)$ & $72.9-92.5$ & $11.3(-1.6$ to 18.9$)$ & 0.09 \\
\hline \multicolumn{7}{|c|}{ Without antiviral medication in viral hepatitis-related cirrhosis $(n=45)^{\mathrm{a}}$} \\
\hline Sensitivity & $100(4 / 4)$ & $39.6-100$ & $25(1 / 4)$ & $1.3-78.1$ & $75(-31$ to 75$)$ & 0.25 \\
\hline Specificity & $95.1(39 / 41)$ & $82.2-99.2$ & $95.1(39 / 41)$ & $82.2-99.2$ & $0(-6.7$ to 6.7$)$ & $>0.99$ \\
\hline
\end{tabular}

US, ultrasonography; LDCT, low-dose computed tomography; BMI, body mass index; CI, confidence interval; LDCT, low-dose CT; US, ultrasonography. ${ }^{\text {a }}$ Eleven participants without viral hepatitis-related cirrhosis (non-HCC:HCC = 10:1) were excluded.

antiviral medication, LDCT tended to show higher sensitivity than US, but it did not reach a statistical significance (Table 4; $p=0.13-0.25$ ).

For detection of very early-stage HCC, the sensitivity of two-phase LDCT was higher than that of US (82.4\% [14/17] vs. 17.7\% [3/17], $p<0.001)$. There was no HCC that was detected only at US. Regarding early-stage HCC, there was no significant difference in sensitivity between two-phase LDCT and US (85.7\% [6/7] vs. 57.1\% [4/7], $p=0.13)$. 
Yoon et al.: HCC Surveillance Using Biannual Two-Phase LDCT and US in a High-Risk Group

Fig. 5. A 53-year old woman without HCC. At US, a 13-mm hypoechoic observation is seen in the right lobe of the liver (arrowheads). No observation was detected at two-phase LDCT and follow-up standard-dose four-phase liver CT (not shown).

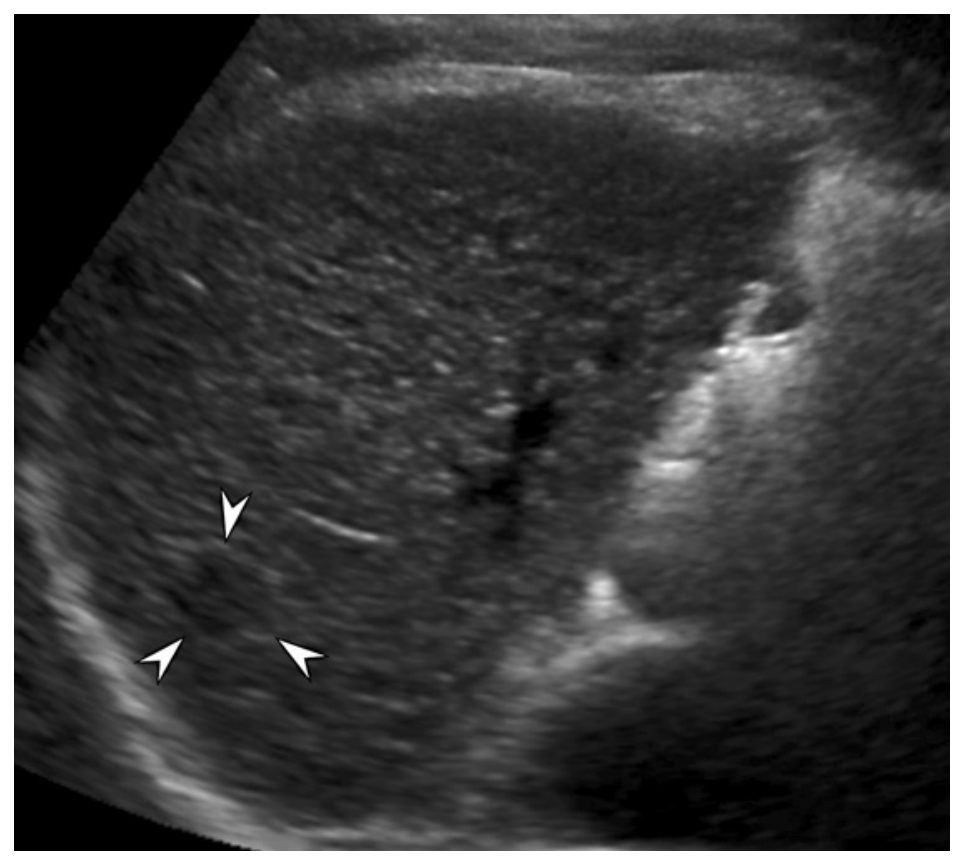

False-Positive Rates for the Two Protocols and Review of Two-Phase LDCT Images

False-positive rates were 4.3\% (5/113) for two-phase LDCT and $12.3 \%(14 / 113)$ for US $(p=0.049)$. At two-phase LDCT, false-positive findings $(n=5)$ included nodular arterioportal shunt $(n=3)$ (which either disappeared or persisted without change during follow-up) and non-APHE observations without interval change on follow-up $(n=2)$. False-positive findings from US included either hypoechoic $(n=13)$ or hyperechoic $(n=1)$ observations with an average size of $14 \mathrm{~mm}$ (range 10-20 mm) (Fig. 5). Observations were not visualized via follow-up standard-dose CT or gadoxetic acid-enhanced MRI $(n=12)$ or seen as non-APHE observations without diffusion restriction and interval change $(n=2)$. There were 4 falsenegative cases for two-phase LDCT: 3 were reported as nodular arterioportal shunt, and 1 was not seen using two-phase LDCT and follow-up.

\section{Radiation Dose}

Participants underwent two-phase LDCT using either $80 \mathrm{kVp}(n=92)$ or $100 \mathrm{kVp}(n=$ 46). The average dose-length product was $527.8 \pm 200.5 \mathrm{mGy}$ in all participants. The estimated effective dose was $7.9 \pm 3.0 \mathrm{mSv}$ on average.

\section{Discussion}

Although regular surveillance for HCC using US has been shown to be related to better overall survival $[28,29]$, the benefit of surveillance is critically affected by the sensitivity of the surveillance modality [19]. In other words, the more sensitive the surveillance modality for detection of HCC, the better the outcome anticipated. In our study, two-phase LDCT was far more sensitive than US for the detection of HCC in participants with a high risk of developing HCC (83.3 vs. $29.2 \%, p<0.001$ ). Moreover, two-phase LDCT also showed significantly higher sensitivity for very early-stage HCC (single, $<2 \mathrm{~cm}$ ) detection than US $(82.4$ vs. $17.7 \%$, $p<0.001$ ). Our study results are consistent with those of a recent study which demonstrated that hepatocyte-specific contrast-enhanced liver MRI yielded better sensitivity than US (84.8 
and $27.3 \%$, respectively) for very early-stage HCC detection [17]. Given that very early-stage HCC shows $80-89.5 \%$ of 5-year overall survival after hepatic resection or radiofrequency ablation [30, 31], we believe that two-phase LDCT can be considered an advantageous option for HCC surveillance in patients with a high risk of developing HCC.

More importantly, two-phase LDCT also demonstrated a significantly higher specificity, PPV, and NPV than US by showing fewer false-positive and false-negative findings. This is clinically important because two-phase LDCT may reduce the prevalence of unnecessary follow-up and additional examination due to false-positive findings at US. A study reports that physical harm resulting from false-positive findings in HCC surveillance, using either US or AFP, was observed in $27.5 \%$ of cases, including one-time or multiple CT or MRI examinations, biopsy, and angiography [32]. Although false-positive findings are an inevitable part of surveillance tests, it would be clinically beneficial if we could achieve sufficient specificity to prevent an unnecessary examination. Based on our study results showing that two-phase LDCT provided both significantly higher sensitivity and a significantly lower false referral rate than US, two-phase LDCT could be more beneficial than US for HCC surveillance in highrisk patients.

In our study, detection of HCC at a very early/early-stage was $29.2 \%$ (7/24) with US. This outcome is somewhat disappointing but consistent with the results both of a meta-analysis reporting 42\% sensitivity for early-stage HCC (95\% CI: 27-58\%) [10] and also those of a prospective cohort study reporting $27.9 \%$ sensitivity for early-stage HCC [17]. Indeed, surveillance failure using US is not uncommon in the literature [17,33-35]. The limited performance of US for early-stage or very early-stage HCC can be explained by the small size of tumors; the limited sonic windows due to patients' body habitus, operators' experience, the inherent blind spot of US, and the underlying distortion of parenchyma in macronodular cirrhosis [33]. In particular, our eligibility criterion (risk index $\geq 2.33$ ) indicates the presence of advanced cirrhosis; and accordingly, there may be an increased risk of a limited sonic window and substantial distortion of liver parenchyma where US may perform unreliably [17]. Our study results suggest that US may not be an appropriate option in some high-risk patients with advanced cirrhosis, because factors that limit the US performance are often not amendable [36], even with CEUS [18].

Another important finding in our study is that our eligibility criteria were able to identify the group with a "very-high" risk of HCC development. Identifying a "very-high" risk group is critical for performing CT as a surveillance modality, as patients with a "very-high" risk of developing HCC may require intensive investigation using an alternative with a higher sensitivity than biannual US. Despite the concerns that a "one-size-fits-all" strategy may not work effectively for HCC surveillance, currently only the Japan Society of Hepatology defines an "extremely-high" risk group with hepatitis B or C cirrhosis for whom a different surveillance test (CT or MRI) and intensity [37] are recommended. Herein, we used the risk index that presumably indicated 5\% of the estimated annual incidence of HCC development based on a previous study [24]. The estimated annual incidence of HCC development was $11.5 \%$ in our study, which was higher than the expected figure. This may be explained via the high incidence of viral hepatitis, especially chronic hepatitis B $(83.3 \%)$ which is a well-known risk factor for HCC, as was reported in a prospective chronic hepatitis B-rich cohort with the same risk index [17]. Based on our observations, we believe that there are patients who have a higher risk of developing HCC among the population who are indicative of undergoing HCC surveillance: a so-called "very-high" risk group, and the risk index we used would be one metric to identify this population. Recent studies using models and individualization of risk factors may help to identify the "very-high" risk group more precisely [38-40].

Although two-phase LDCT performed better than US for early HCC detection, CT remains controversial as a surveillance modality because of its cost-effectiveness, radiation hazard, 
and potential adverse effect of contrast media. Strategies related to minimizing the radiation dose and identifying "very-high" risk groups will be the key to resolving the aforementioned issues. In our study, we used low kVp scans and limited scan coverage to the liver [41], additionally obtaining only two phases [22] to minimize the radiation dose while managing diagnostic performance. We also excluded those with renal dysfunction or contrast media hypersensitivity in our selected population. However, contrast media-induced nephrotoxicity is rare in patients with normal or mild-to-moderate renal dysfunction [42, 43]. Further, contrast media-induced hypersensitivity is mild in most patients, and mild hypersensitivity reaction can be prevented with changing types of contrast media with or without medication of antihistamines [44]. Ultimately, concerns about exposure to radiation and contrast media should be balanced with consideration of the potential benefit of CT [45]. Two-phase LDCT may not be feasible for all patients with cirrhosis, because of their variable risks of developing HCC. But a "very-high" risk population, especially those in their old age, can benefit from two-phase LDCT because of reduced concern about radiation exposure compared with young patients and higher sensitivity of two-phase LDCT for detecting HCC [46]. Thus, two-phase LDCT would be a reasonable alternative for HCC surveillance in a selected population. Regarding cost-effectiveness, identifying a "very-high" risk group is a critical point, because such alternatives with higher sensitivity and cost than the standard of care can be justified only when the disease has a high prevalence. Studies have reported that HCC surveillance using CT or MRI can be more cost-effective than US in the case of patients with a high risk of HCC development $[36,47,48]$. A recent cost-effectiveness analysis study reported that with increased HCC incidence, even biannual gadoxetic acid-enhanced liver MRI could be more cost-effective than US [48]. Compared with gadoxetic acid-enhanced MRI, two-phase LDCT has the disadvantage of radiation exposure, yet has the advantages of cost, facility accessibility, and reduced scan time that may influence patients' compliance. Although CEUS has also drawn attention in relation to HCC surveillance [49] because of its low cost, a recent prospective study using CEUS failed to improve the detection of early-stage HCC [17]. Thus, CEUS perhaps would not be the best approach for a group with a "very-high" risk of HCC development. We believe that the high incidence of HCC development, the average age of our study population, and reduced radiation dose would justify the use of two-phase LDCT as a selectively used alternative surveillance test. In addition, standalone two-phase LDCT covers the whole liver and when performed for surveillance may be provided at a lower cost than standard-dose four-phase CT, in a way similar to abbreviated liver MRI [47]. Alternate use of US and two-phase LDCT should be evaluated to further reduce radiation exposure and cost in future studies.

Our study has several limitations. First, the sample size is relatively small. Second, the diagnoses of HCC were made based on imaging because of current clinical practice. Third, we did not analyze the cost-effectiveness of the two modalities because of the limited number of study participants. However, as we mentioned earlier, studies report that biannual gadoxetic acid-enhanced MRI or CT can be more cost-effective than US in a high-risk group [47, 48]. Fourth, the results of the two-phase LDCT at the previous round were available to radiologists who performed US at the next round. While this may have caused some bias, we think that blinding colleagues to participants' prior examinations is not ethically acceptable because it is not in the patients' best interest. Fifth, the $\mathrm{kVp}$ varied according to participants' weight. Further detailed clarification of $\mathrm{kVp}$ selection or automatic $\mathrm{kV}$ selection [50] would be helpful to further reduce the radiation dose. Sixth, most of our study population had viral hepatitisdriven liver cirrhosis; and, therefore, our results are not applicable to other cirrhotic patient populations with different etiologies. Seventh, we did not exclude Child-Pugh C patients, although the included number $(n=2)$ was small. Eighth, we did not include AFP for US surveillance. This is because our study aimed to compare two imaging modalities for detecting HCC. Further, elevated AFP level was observed in only 2 participants with HCC in our study. Because 
our study sample size is small and a different cut-off value of AFP has been suggested for surveillance in some studies, the role of AFP should be investigated in a large study population [51, 52]. Last, we did not analyze the relationship between early detection of HCC and survival gain, which may be the ultimate goal of HCC surveillance. Further studies with a larger study population are warranted.

In conclusion, biannual two-phase LDCT surveillance showed a significantly higher detection rate for very early/early-stage HCC and a lower frequent false-positive reporting rate than biannual US.

\section{Acknowledgments}

The authors thank Benjamin Latimer, MA, for his editorial assistance.

\section{Statement of Ethics}

This prospective single-arm study was approved by the Institutional Review Board of Seoul National University Hospital. All protocols were carried out in accordance with the latest Helsinki Declaration (revised 2013). Written informed consent was obtained from each participant (NCT02675920).

\section{Conflict of Interest Statement}

Jeong Hee Yoon: activities related to the present article: disclosed no relevant relationship. Activities not related to the present article: the author previously received grants from Bayer; and personal fees from Philips Healthcare, Samsung Electronics, and Bayer.

Jeong Min Lee: activities related to the present article: disclosed no relevant relationship. Activities not related to the present article: the author previously received nonfinancial technical support from Siemens Healthineers and Philips Healthcare; grants from Dongseo Medical, CMS, Acuzen, Starmed, RF Medical, and Bayer; and personal fees from Bayer Healthcare for lectures.

Ijin Joo, Dong Ho Lee, Su Joa Ahn, Ju Hyun Jeon, Seung-taak Kim, Eun Ju Cho, Su Jong Yu, and Yoon Jun Kim: disclosed no relevant relationship.

Jeong-Hoon Lee: activities related to the present article: disclosed no relevant relationship. Activities not related to the present article: the author previously received lecture fees from GreenCross Cell, Daewoong Pharmaceuticals, and Gilead Korea.

Jung-Hwan Yoon: activities related to the present article: disclosed no relevant relationship. Activities not related to the present article: the author previously received research grants from Bayer, Bukwang Pharmaceuticals, and Daewoong Pharmaceuticals.

\section{Funding Sources}

This study was financially supported by Acuzen and Reyon Pharmaceutical equally. The funding sources had no other roles in preparation of data or the manuscript.

\section{Author Contributions}

All authors had access to the study data and reviewed and approved the final manuscript. Study concept/ design: all authors. Data acquisition: Jeong Hee Yoon, Jeong Min Lee, Dong Ho Lee, Ijin Joo, Ju Hyun Jeon, Su Joa Ahn, and Seung-taek Kim. Data analysis: Jeong Hee Yoon and Jeong Min Lee. Data interpretation: all authors. Drafting of the manuscript: Jeong Hee Yoon and Jeong Min Lee. Critical revision of the manuscript for important intellectual content: all authors. Statistical analysis: statistical analysis was conducted by the Medical Research Collaborating Center in Seoul National University Hospital according to the proposed statistical analysis plan, under the supervision of Jeong Hee Yoon and Jeong Min Lee. Obtained funding: Jeong Min Lee. Study supervision: Jeong Min Lee. 


\begin{tabular}{l|l|}
\hline Liver Cancer 2020;9:503-517 \\
\hline DOI: 10.1159/000506834 & $\begin{array}{l}\text { @ 2020 The Author(s). Published by S. Karger AG, Basel } \\
\text { www.karger.com/lic }\end{array}$ \\
\hline
\end{tabular}

Yoon et al.: HCC Surveillance Using Biannual Two-Phase LDCT and US in a High-Risk Group

\section{References}

1 Bray F, Ferlay J, Soerjomataram I, Siegel RL, Torre LA, Jemal A. Global cancer statistics 2018: GLOBOCAN estimates of incidence and mortality worldwide for 36 cancers in 185 countries. CA Cancer J Clin. 2018 Nov; 68(6):394-424.

2 Fujiwara N, Friedman SL, Goossens N, Hoshida Y. Risk factors and prevention of hepatocellular carcinoma in the era of precision medicine. J Hepatol. 2018 Mar;68(3):526-49.

3 Serper M, Taddei TH, Mehta R, D’Addeo K, Dai F, Aytaman A, et al.; VOCAL Study Group. Association of Provider Specialty and Multidisciplinary Care With Hepatocellular Carcinoma Treatment and Mortality. Gastroenterology. 2017 Jun;152(8):1954-64.

4 Kim BH, Lim YS, Kim EY, Kong HJ, Won YJ, Han S, et al. Temporal improvement in survival of patients with hepatocellular carcinoma in a hepatitis B virus-endemic population. J Gastroenterol Hepatol. 2018 Feb;33(2):475-83.

5 Bertuccio P, Turati F, Carioli G, Rodriguez T, La Vecchia C, Malvezzi M, et al. Global trends and predictions in hepatocellular carcinoma mortality. J Hepatol. 2017 Aug;67(2):302-9.

6 Fitzmorris P, Singal AK. Surveillance and Diagnosis of Hepatocellular Carcinoma. Gastroenterol Hepatol (N Y). 2015 Jan;11(1):38-46.

7 Galle PR, Forner A, Llovet JM, Mazzaferro V, Piscaglia F, Raoul JL, et al.; European Association for the Study of the Liver. EASL Clinical Practice Guidelines: Management of hepatocellular carcinoma. J Hepatol. 2018;69(1): 182-236.

8 Heimbach JK, Kulik LM, Finn RS, Sirlin CB, Abecassis MM, Roberts LR, et al. AASLD guidelines for the treatment of hepatocellular carcinoma. Hepatology. 2018 Jan;67(1):358-80.

9 Omata M, Cheng AL, Kokudo N, Kudo M, Lee JM, Jia J, et al. Asia-Pacific clinical practice guidelines on the management of hepatocellular carcinoma: a 2017 update. Hepatol Int. 2017 Jul;11(4):317-70.

10 Tzartzeva K, Obi J, Rich NE, Parikh ND, Marrero JA, Yopp A, Waljee AK, Singal AG: Surveillance Imaging and Alpha Fetoprotein for Early Detection of Hepatocellular Carcinoma in Patients With Cirrhosis: A Meta-analysis. Gastroenterology. 2018 May;154(6):1706-18.e1.

11 Singal A, Volk ML, Waljee A, Salgia R, Higgins P, Rogers MA, et al. Meta-analysis: surveillance with ultrasound for early-stage hepatocellular carcinoma in patients with cirrhosis. Aliment Pharmacol Ther. 2009 Jul;30(1):37-47.

12 Kim BR, Lee JM, Lee DH, Yoon JH, Hur BY, Suh KS, et al. Diagnostic Performance of Gadoxetic Acid-enhanced Liver MR Imaging versus Multidetector CT in the Detection of Dysplastic Nodules and Early Hepatocellular Carcinoma. Radiology. 2017 Oct;285(1):134-46.

13 Liu WC, Lim JH, Park CK, Kim MJ, Kim SH, Lee SJ, et al. Poor sensitivity of sonography in detection of hepatocellular carcinoma in advanced liver cirrhosis: accuracy of pretransplantation sonography in 118 patients. Eur Radiol. 2003 Jul;13(7):1693-8.

14 Yu NC, Chaudhari V, Raman SS, Lassman C, Tong MJ, Busuttil RW, Lu DS: CT and MRI improve detection of hepatocellular carcinoma, compared with ultrasound alone, in patients with cirrhosis. Clin Gastroenterol Hepatol. 2011;9:161-7.

15 Andersson KL, Salomon JA, Goldie SJ, Chung RT. Cost effectiveness of alternative surveillance strategies for hepatocellular carcinoma in patients with cirrhosis. Clin Gastroenterol Hepatol. 2008 Dec;6(12):1418-24.

16 Pocha C, Dieperink E, McMaken KA, Knott A, Thuras P, Ho SB. Surveillance for hepatocellular cancer with ultrasonography vs. computed tomography— a randomised study. Aliment Pharmacol Ther. 2013 Aug;38(3):303-12.

17 Kim SY, An J, Lim YS, Han S, Lee JY, Byun JH, et al. MRI With Liver-Specific Contrast for Surveillance of Patients With Cirrhosis at High Risk of Hepatocellular Carcinoma. JAMA Oncol. 2017 Apr;3(4):456-63.

18 Park JH, Park MS, Lee SJ, Jeong WK, Lee JY, Park MJ, et al. Contrast-enhanced US with Perfluorobutane for Hepatocellular Carcinoma Surveillance: A Multicenter Diagnostic Trial (SCAN). Radiology. 2019 Sep;292(3): 638-46.

19 Yang JD, Mannalithara A, Piscitello AJ, Kisiel JB, Gores GJ, Roberts LR, et al. Impact of surveillance for hepatocellular carcinoma on survival in patients with compensated cirrhosis. Hepatology. 2018 Jul;68(1):78-88.

20 Goldman AR, Maldjian PD. Reducing radiation dose in body CT: a practical approach to optimizing CT protocols. AJR Am J Roentgenol. 2013 Apr;200(4):748-54.

21 McCollough CH, Primak AN, Braun N, Kofler J, Yu L, Christner J. Strategies for reducing radiation dose in CT. Radiol Clin North Am. 2009 Jan;47(1):27-40.

22 Jang HJ, Kim TK, Khalili K, Yazdi L, Menezes R, Park SH, et al. Characterization of 1-to 2-cm liver nodules detected on hcc surveillance ultrasound according to the criteria of the American Association for the Study of Liver Disease: is quadriphasic CT necessary? AJR Am J Roentgenol. 2013 Aug;201(2):314-21.

23 Marrero JA, Kulik LM, Sirlin CB, Zhu AX, Finn RS, Abecassis MM, et al. Diagnosis, Staging, and Management of Hepatocellular Carcinoma: 2018 Practice Guidance by the American Association for the Study of Liver Diseases. Hepatology. 2018 Aug; 68(2):723-50.

24 Velázquez RF, Rodríguez M, Navascués CA, Linares A, Pérez R, Sotorríos NG, et al. Prospective analysis of risk factors for hepatocellular carcinoma in patients with liver cirrhosis. Hepatology. 2003 Mar;37(3):520-7.

25 The 2007 Recommendations of the International Commission on Radiological Protection. ICRP publication 103. Ann ICRP. 2007;37(2-4):1-332.

26 American College of Radiology. Liver Imaging Reporting and Data System version 2014. Reston, VA: ACR; 2016.

27 European Association for the Study of The Liver; European Organisation for Research and Treatment of Cance. EASL-EORTC clinical practice guidelines: management of hepatocellular carcinoma. J Hepatol. 2012;56(4): 908-43. 


\begin{tabular}{|c|c|}
\hline \multicolumn{2}{|l|}{ Liver Cancer 2020;9:503-517 } \\
\hline DOI: $10.1159 / 000506834$ & $\begin{array}{l}\text { (c) } 2020 \text { The Author(s). Published by S. Karger AG, Basel } \\
\text { www.karger.com/lic }\end{array}$ \\
\hline
\end{tabular}

Yoon et al.: HCC Surveillance Using Biannual Two-Phase LDCT and US in a High-Risk Group

28 Yang B, Zhang B, Xu Y, Wang W, Shen Y, Zhang A, et al. Prospective study of early detection for primary liver cancer. J Cancer Res Clin Oncol. 1997;123(6):357-60.

29 Zhang BH, Yang BH, Tang ZY. Randomized controlled trial of screening for hepatocellular carcinoma. J Cancer Res Clin Oncol. 2004 Jul;130(7):417-22.

30 Tsilimigras DI, Bagante F, Sahara K, Moris D, Hyer JM, Wu L, et al. Prognosis After Resection of Barcelona Clinic Liver Cancer (BCLC) Stage 0, A, and B Hepatocellular Carcinoma: A Comprehensive Assessment of the Current BCLC Classification. Ann Surg Oncol. 2019 Oct;26(11):3693-700.

31 Chu HH, Kim JH, Kim PN, Kim SY, Lim YS, Park SH, Ko HK, Lee SG: Surgical resection versus radiofrequency ablation very early-stage HCC ( $\leq 2$ cm Single HCC): A propensity score analysis. Liver Int. 2019;39:2397-407.

32 Atiq 0, Tiro J, Yopp AC, Muffler A, Marrero JA, Parikh ND, et al. An assessment of benefits and harms of hepatocellular carcinoma surveillance in patients with cirrhosis. Hepatology. 2017 Apr;65(4):1196-205.

33 Sinn DH, Yi J, Choi MS, Choi D, Gwak GY, Paik YH, et al. Incidence and risk factors for surveillance failure in patients with regular hepatocellular carcinoma surveillance. Hepatol Int. 2013 Oct;7(4):1010-8.

34 Singal AG, Nehra M, Adams-Huet B, Yopp AC, Tiro JA, Marrero JA, et al. Detection of hepatocellular carcinoma at advanced stages among patients in the HALT-C trial: where did surveillance fail? Am J Gastroenterol. 2013 Mar;108(3):425-32.

35 Mancebo A, Varela M, González-Diéguez ML, Navascués CA, Cadahía V, Mesa-Álvarez A, et al. Incidence and risk factors associated with hepatocellular carcinoma surveillance failure. J Gastroenterol Hepatol. 2018 Aug; 33(8):1524-9.

36 Goossens N, Singal AG, King LY, Andersson KL, Fuchs BC, Besa C, et al. Cost-Effectiveness of Risk Score-Stratified Hepatocellular Carcinoma Screening in Patients with Cirrhosis. Clin Transl Gastroenterol. 2017 Jun; 8(6):e101.

37 Kudo M, Matsui O, Izumi N, Iijima H, Kadoya M, Imai Y; Liver Cancer Study Group of Japan. Surveillance and diagnostic algorithm for hepatocellular carcinoma proposed by the Liver Cancer Study Group of Japan: 2014 update. Oncology. 2014;87(s1 Suppl 1):7-21.

38 Papatheodoridis G, Dalekos G, Sypsa V, Yurdaydin C, Buti M, Goulis J, et al. PAGE-B predicts the risk of developing hepatocellular carcinoma in Caucasians with chronic hepatitis B on 5-year antiviral therapy. J Hepatol. 2016 Apr;64(4):800-6.

39 Goossens N, Bian CB, Hoshida Y. Tailored algorithms for hepatocellular carcinoma surveillance: is one-sizefits-all strategy outdated? Curr Hepatol Rep. 2017 Mar;16(1):64-71.

40 Ganne-Carrié N, Layese R, Bourcier V, Cagnot C, Marcellin P, Guyader D, et al.; ANRS C012 CirVir Study Group. Nomogram for individualized prediction of hepatocellular carcinoma occurrence in hepatitis $\mathrm{C}$ virus cirrhosis (ANRS C012 CirVir). Hepatology. 2016 Oct;64(4):1136-47.

41 Yu MH, Lee JM, Yoon JH, Baek JH, Han JK, Choi BI, et al. Low tube voltage intermediate tube current liver MDCT: sinogram-affirmed iterative reconstruction algorithm for detection of hypervascular hepatocellular carcinoma. AJR Am J Roentgenol. 2013 Jul;201(1):23-32.

42 Nijssen EC, Nelemans PJ, Rennenberg RJ, van Ommen V, Wildberger JE. Prophylactic Intravenous Hydration to Protect Renal Function From Intravascular Iodinated Contrast Material (AMACING): Long-term Results of a Prospective, Randomised, Controlled Trial. EClinicalMedicine. 2018 Nov;4-5:109-16.

43 Nijssen EC, Rennenberg RJ, Nelemans PJ, Essers BA, Janssen MM, Vermeeren MA, et al. Prophylactic hydration to protect renal function from intravascular iodinated contrast material in patients at high risk of contrastinduced nephropathy (AMACING): a prospective, randomised, phase 3, controlled, open-label, non-inferiority trial. Lancet. 2017 Apr;389(10076):1312-22.

44 Park SJ, Kang DY, Sohn KH, Yoon SH, Lee W, Choi YH, et al. Immediate Mild Reactions to CT with Iodinated Contrast Media: Strategy of Contrast Media Readministration without Corticosteroids. Radiology. 2018 Sep; 288(3):710-6.

45 Rampinelli C, De Marco P, Origgi D, Maisonneuve P, Casiraghi M, Veronesi G, et al. Exposure to low dose computed tomography for lung cancer screening and risk of cancer: secondary analysis of trial data and riskbenefit analysis. BMJ. 2017 Feb;356:j347.

46 Park JW, Chen M, Colombo M, Roberts LR, Schwartz M, Chen PJ, Kudo M, Johnson P, Wagner S, Orsini LS, Sherman M: Global patterns of hepatocellular carcinoma management from diagnosis to death: the BRIDGE Study. Liver Int. 2015;35:2155-66.

47 Lima PH, Fan B, Bérubé J, Cerny M, Olivié D, Giard JM, et al. Cost-Utility Analysis of Imaging for Surveillance and Diagnosis of Hepatocellular Carcinoma. AJR Am J Roentgenol. 2019 Apr;213(1):1-9.

48 Kim HL, An J, Park JA, Park SH, Lim YS, Lee EK. Magnetic Resonance Imaging Is Cost-Effective for Hepatocellular Carcinoma Surveillance in High-Risk Patients With Cirrhosis. Hepatology. 2019 Apr;69(4):1599-613.

49 Li J, Wang J, Yu S, Yuan G, He S. Hepatocellular Carcinoma Surveillance in High-Risk Patients with Cirrhosis: Contrast-Enhanced Ultrasound May Be a Choice. Hepatology. 2019.

50 Lee KH, Lee JM, Moon SK, Baek JH, Park JH, Flohr TG, et al. Attenuation-based automatic tube voltage selection and tube current modulation for dose reduction at contrast-enhanced liver CT. Radiology. 2012 Nov;265(2):437-47.

51 Choi J, Kim GA, Han S, Lee W, Chun S, Lim YS. Longitudinal Assessment of Three Serum Biomarkers to Detect Very Early-Stage Hepatocellular Carcinoma. Hepatology. 2020 Jan;71(1):392.

52 Kim MN, Kim BK, Kim SU, Park JY, Ahn SH, Han KH, et al. Longitudinal assessment of alpha-fetoprotein for early detection of hepatocellular carcinoma in patients with cirrhosis. Scand J Gastroenterol. 2019 Oct;54(10): 1283-90. 